\title{
Perfil dos pacientes submetidos à neurocirurgia para tratamento de aneurismas intracranianos
}

\author{
Profile of patients submitted to neurosurgical procedures for intracranial aneurysm
}

\author{
Lucas Bezerra dos Santos ${ }^{1}$, Camila Waters ${ }^{2}$
}

\section{Resumo}

Objetivo: Identificar o perfil epidemiológico, clínico e desfecho dos pacientes submetidos à neurocirurgia para tratamento de aneurismas intracranianos. Métodos: Pesquisa retrospectiva, com dados de prontuários dos pacientes com idade superior a 18 anos, submetidos à tratamento neurocirúrgico para clipagem de aneurisma intracraniano no período de 01/01/2015 a 01/07/2015, após aprovação pelo CEP (32959114.6.0000.5479). Resultados: Dos 28 pacientes incluídos no estudo, prevaleceu o gênero feminino em 64,3\%, com média de idade de 49,6 anos, variando de 19 a 66 anos. A maioria (60,7\%) chegou à instituição sendo transferido de outro serviço hospitalar. Permaneceram em média 19 dias internados no hospital, sendo que 39,3\% permaneceram de 8 a 11 dias. A média de internação na UTI foi de 10,5 dias. A maioria (35,7\%) apresentou uma escala de Hunt-Hess de valor II e na escala de Fisher prevaleceu a pontuação IV em $46,4 \%$ dos pacientes. Predominaram aneurismas únicos $(75,0 \%)$, rotos $(89,3 \%)$, acometendo a circulação anterior $(82,1 \%)$, mais comum na artéria comunicante anterior $(32,1 \%) e$ artéria cerebral média $(28,7 \%)$. Na maioria dos pacientes $(60,7 \%)$ a abordagem cirúrgica foi em até três dias desde a chegada ao hospital, sendo cirurgias sem intercorrências $(89,3 \%)$, com uma média de duração de 4 h37, variando de 3h20 a 9h10. Complicações pós-operatórias ocorreram em $46,4 \%$ dos pacientes, $67,9 \%$ evoluíram com sequelas e a mortalidade foi de 17,9\%. Conclusões: Prevaleceram pacientes adultos jovens, do gênero feminino, que chegaram

1. Graduado em Enfermagem pela Faculdade de Ciências Médicas da Santa Casa de São Paulo

2. Professora Assistente da Faculdade de Ciências Médicas da Santa Casa de São Paulo - Curso de Graduação em Enfermagem

Trabalho realizado: Faculdade de Ciências Médicas da Santa Casa de São Paulo - Curso de Graduação em Enfermagem Declaramos não haver conflito de interesse

Endereço para correspondência: Lucas Bezerra dos Santos. Rua Miguel Fernandes Maldonado, $n^{\circ} 507$ - Jardim Santa Rita - 07143-222 - Guarulhos - SP - Brasil. E-mail: lucas_lbs5@hotmail.com à Instituição transferidos de outro hospital, com aneurismas únicos, rotos, em circulação anterior, evoluindo sem sequelas e com uma baixa mortalidade.

Descritores: Perfil epidemiológico, Neurocirurgia, Aneurisma intracraniano, Aneurisma cerebral

\begin{abstract}
Objective: To identify the epidemiological, clinical and outcome profiles of patients undergoing neurosurgeries for the treatment of intracranial aneurysms. Methods: Retrospective study, with data from medical records of patients over 18 years of age, submitted to neurosurgical treatments for intracranial aneurysm clippings from 01/01/2015 to 07/01/2015, after Institutional Board approval (32959114.6.0000.5479). Results: Of the 28 patients included in this study, female gender prevailed in $64,3 \%$, with a mean age of 49.6 years, ranging from 19 to 66 years of age. The majority $(60,7 \%)$ arrived at the institution being transferred from another hospital service. Patients remained an average of 19 days in the hospital, being that $39.3 \%$ remained from 8 to 11 days. The mean Intensive Care Unit stay was 10.5 days. The majority (35,7\%) presented a Hunt-Hess scale of II value and in the Fisher scale, the IV score prevailed in $46,4 \%$ of the patients. There was a predominance of single aneurysms $(75,0 \%)$, ruptured $(89,3 \%)$, anterior circulation $(82,1 \%)$, anterior communicating artery $(32,1 \%)$ were the most common followed by middle cerebral artery aneurisms $(28,7 \%)$. In the majority of patients $(60,7 \%)$, surgical approach was performed within three days of Hospital arrival. Surgeries were uneventful in $89,3 \%$ of the cases, with a mean duration of $4437 \mathrm{~min}$, varying from 3:20 to 9:10 hours. Postoperative complications occurred in $46,4 \%$ of the patients, $67,9 \%$ had sequelae, and mortality was 17,9\%. Conclusions: Prevalence of young adult female patients who arrived at the institution transferred from another hospital, with single aneurysms, ruptured, in the anterior circulation, evolving without sequel and with a low mortality.
\end{abstract}

Keywords: Health profile, Neurosurgery, Intracranial aneurysm, Brain aneurysm 


\section{Introdução}

Aneurismas intracranianos são dilatações, localizadas e anormais, na parede das artérias cerebrais ${ }^{(1)}$ com risco potencial para ruptura ${ }^{(2)}$ causando hemorragia aguda ${ }^{(3)}$. Aproximadamente $75,0 \%$ de todas as hemorragias subaracnóideas espontâneas são causadas pela ruptura de um aneurisma sacular ${ }^{(4)}$. A incidência aneurismática, segundo achados em autópsias, varia entre 0,2 a $9,9 \%$ da população geral, e se estima que aproximadamente 1,5 a $2 \%$ dos vivos apresentem aneurismas intracranianos ${ }^{(5-6)}$. No Brasil, cerca de $12 \%$ dos indivíduos acometidos morrem antes de obter atendimento médico e cerca de $40 \%$ morrem em até um mês após o incidente ${ }^{(7)}$. Cerca de $80 \%$ dos casos ocorre entre 40 e 65 anos de idade, sendo que a população feminina é mais afetada ${ }^{(3,8)}$, especialmente após a menopausa ${ }^{(9)}$. Outros fatores predisponentes são o etilismo e tabagismo, hipertensão arterial sistêmica, diabetes mellitus, dislipidemias ${ }^{(3)}$ e o uso de estrogênios, cocaína, cafeína e outras drogas simpaticomiméticas ${ }^{(6)}$. Inicialmente o objetivo do tratamento é estabilização clínica e eliminação do risco de novo sangramento, e se dá através da exclusão do saco aneurismático da circulação intracraniana preservando a artéria mãe e seus ramos ${ }^{(6)}$. A abordagem clássica consiste na clipagem neurocirúrgica ${ }^{(10)}$, que é a colocação de um clipe metálico entre o vaso normal e colo aneurismático, excluindo assim a circulação sanguínea local, evitando o ressangramento nos casos de ruptura aneurismática ${ }^{(11)}$. O serviço de neurocirurgia do Hospital da Irmandade da Santa Casa de Misericórdia de São Paulo, anualmente, possui elevada demanda de atendimentos com necessidade de tratamento neurocirúrgico ${ }^{(12)}$. Os pacientes com aneurismas intracranianos rotos apresentam diversas especificidades, uma elevada morbimortalidade, requerendo uma assistência de urgência e de forma individualizada ${ }^{(13)}$. A intensidade da hemorragia inicial, a ocorrência de vasoespasmo e o ressangramento são os fatores mais importantes que determinam a morbimortalidade por ruptura de aneurisma intracraniano ${ }^{(4)}$.

Objetivo: Identificar o perfil epidemiológico, clínico e desfecho dos pacientes submetidos à neurocirurgia para tratamento de aneurismas intracranianos.

\section{Material e Métodos}

Pesquisa retrospectiva, descritiva e com análise quantitativa dos dados, desenvolvida no Serviço de Arquivo Médico Estatístico (SAME) do Hospital Central da Irmandade da Santa Casa de Misericórdia de São Paulo (ISCMSP). Selecionados prontuários de pacientes com idade superior a 18 anos, submetidos a tratamento neurocirúrgico para clipagem de aneurisma intracraniano no período de 01/01/2015 a 01/07/2015. Excluídos prontuários indisponíveis para consulta no momento e pacientes submetidos a mais do que uma abordagem neurocirúrgica para clipagem de aneurisma cerebral no período estipulado para o estudo. Projeto aprovado pelo Comitê de Ética em Pesquisa (CEP) da instituição (32959114.6.0000.5479). Os resultados foram apresentados de forma descritiva e em percentual, e apresentados em tabelas e figuras.

\section{Resultados}

Após aprovação do CEP da instituição foi solicitado ao departamento de análise de sistemas a seleção dos pacientes que foram submetidos a qualquer intervenção neurocirúrgica no período de 01/01/2015 a 01/07/2015. O Instituto de Pesquisa da Instituição autorizou a consulta a esses prontuários, que ocorreu entre os dias 21 de março a 10 de maio de 2016. Foram consultados dez prontuários por dia, sendo que dos 109 prontuários consultados, foram selecionados 28 prontuários de pacientes submetidos à cirurgia para a clipagem de aneurisma intracraniano no Hospital Central da ISCMSP.

Tabela 1

Perfil epidemiológico dos pacientes submetidos a tratamento neurocirúrgico para clipagem de aneurisma intracraniano. São Paulo. Jan-Jul, 2015.

\begin{tabular}{lcc}
\multicolumn{1}{c}{ Variável } & $N$ & $\%$ \\
Gênero & 18 & 64,3 \\
Feminino & 10 & 35,7 \\
Masculino & & \\
Faixa etária (anos) & 02 & 7,1 \\
18-28 & 03 & 10,7 \\
$29-39$ & 08 & 28,6 \\
$40-50$ & 10 & 35,7 \\
51-61 & 05 & 17,9 \\
62-72 & & \\
Cor & 20 & 71,4 \\
Branca & 07 & 25,0 \\
Parda & 01 & 3,6 \\
Preta & & \\
Procedência & & $\mathbf{1 0 0 , 0}$ \\
Transferência de outro & 17 & 28,6 \\
serviço & & 10,7 \\
Residência & 08 & \\
Serviço de atendimento & 03 & \\
móvel de urgência & & \\
\hline Total & & \\
\hline & & \\
\hline
\end{tabular}




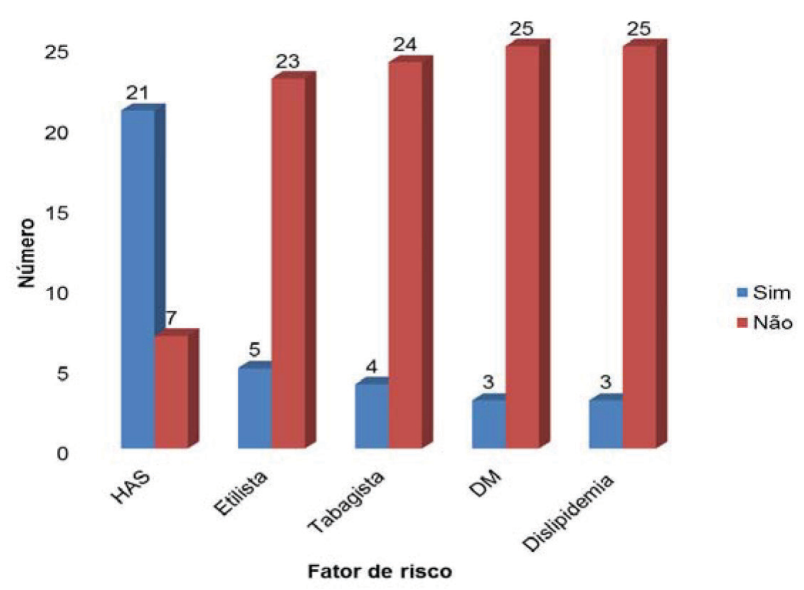

Figura 1 - Figura em barras da distribuição dos pacientes submetidos a tratamento neurocirúrgico para clipagem de aneurisma intracraniano, segundo a presença de fatores de risco. São Paulo. Jan-Jul, 2015.

\section{Tabela 2}

Dados de identificação do procedimento cirúrgico de pacientes submetidos a tratamento neurocirúrgico para clipagem de aneurisma intracraniano. São Paulo. JanJul, 2015.

\section{Variável}

\begin{tabular}{|c|c|c|}
\hline Variável & $N$ & $\%$ \\
\hline \multicolumn{3}{|c|}{$\begin{array}{l}\text { Intervalo de dias entre a admissão hospitalar e a } \\
\text { intervenção neurocirúrgica (dias) }\end{array}$} \\
\hline 0 a 3 & 17 & 60,7 \\
\hline 4 a 7 & 10 & 35,7 \\
\hline$\geq 8$ & 01 & 3,6 \\
\hline
\end{tabular}

Duração do procedimento cirúrgico (horas)

$\begin{array}{lcc}\text { Até 04h00 } & 11 & 39,3 \\ \text { 04h01-05h00 } & 10 & 35,7 \\ \text { 05h01- 06h00 } & 06 & 21,4 \\ \geq 06 h 01 & 01 & 3,6\end{array}$

Complicações pós-operatórias

\begin{tabular}{lcc} 
Não & 15 & 53,6 \\
Sim & 13 & 46,4 \\
Sequelas & & \\
Sim & 19 & 67,9 \\
Não & 09 & 32,1 \\
\hline Total & 28 & 100,0 \\
\hline
\end{tabular}

\section{Discussão}

Os dados obtidos nos mostram que $64,3 \%$ ocorreram em mulheres, corroborando com a literatura científica disponível, a qual indica que tal sexo é mais afetado $^{(4,14-15)}$, na proporção de 3:2(8). Uma hipótese que justifica tal fato pode ser o declínio da produção de hormônios sexuais femininos após a menopausa com

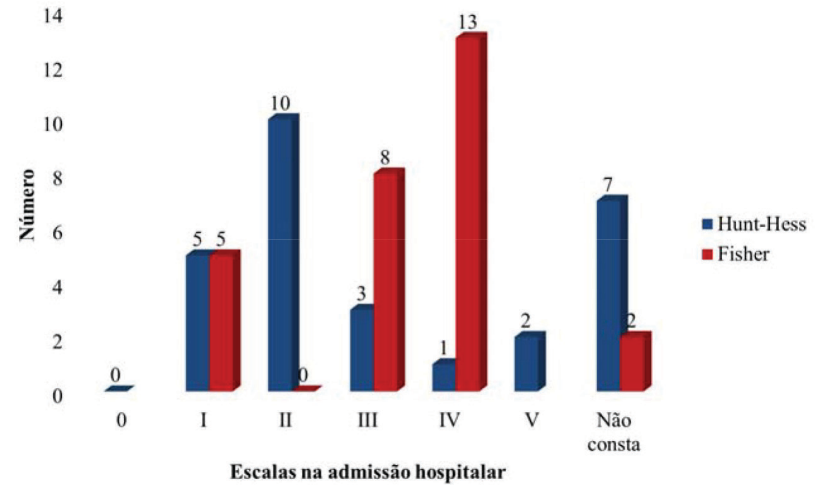

Figura 2 - Figura em barras da distribuição dos pacientes submetidos a tratamento neurocirúrgico para clipagem de aneurisma intracraniano, segundo as escalas de Hunt-Hess e Fisher na admissão hospitalar. São Paulo. Jan-Jul, 2015.

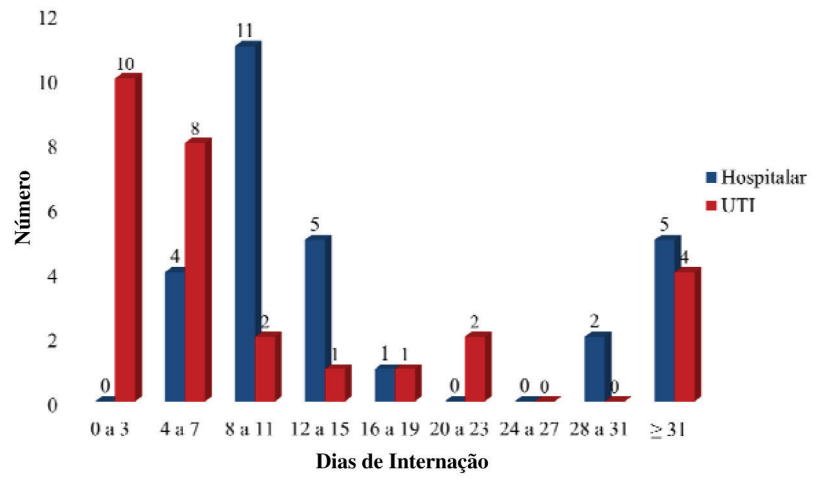

Figura 3 - Figura em barras da distribuição dos pacientes submetidos a tratamento neurocirúrgico para clipagem de aneurisma intracraniano, segundo os dias de internação hospitalar e na UTI. São Paulo. Jan-Jul, 2015.

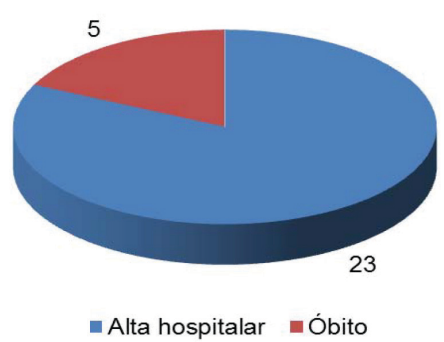

Figura 4 - Figura em pizza da distribuição dos pacientes submetidos a tratamento neurocirúrgico para clipagem de aneurisma intracraniano, segundo o desfecho clínico. São Paulo. Jan-Jul, 2015.

posterior inibição da formação de colágeno o que pode afetar os vasos cerebrais e propiciar o aparecimento de aneurismas ${ }^{(16-18)}$.

Da amostra submetida à cirurgia de clipagem de aneurisma intracraniano, a média geral de idade foi de 49,6 anos, e a faixa etária mais representativa se encontrava entre 40 e 61 anos (64,3\%). Um estudo 
realizado na mesma Instituição avaliou 253 pacientes com hemorragia subaracnóidea espontânea, no período de 1991 a 1996 e encontrou uma média de idade de $48 \operatorname{anos}^{(4)}$, semelhante à encontrada no nosso estudo. Segundo Passos, há ocorrência e ruptura aneurismática em qualquer faixa etária, contudo, de forma mais frequente entre 40 e 65 anos de idade, o que vai de encontro aos dados obtidos no estudo $^{(8)}$.

Segundo a nota informativa 131/2015, do Ministério da Saúde - elaborada pela Coordenação Geral de Média e Alta Complexidade da Secretaria de Atenção à Saúde, no Brasil, existem atualmente 27 hospitais habilitados como Unidade de Assistência ou Centro de Referência de Alta Complexidade em Neurologia e Neurocirurgia, sendo a Santa Casa de Misericórdia de São Paulo um deles ${ }^{(19)}$. Dessa forma, com relação à procedência dos pacientes justifica-se a maior prevalência das transferências de outras unidades de serviços de saúde como maioria, que foi constatado em $60,7 \%$ dos casos.

A média de internação hospitalar dos indivíduos estudados foi de 19 dias. Para os pacientes que obtiveram alta hospitalar, houve variação entre cinco e 80 dias tendo em média 17,08 dias de internação. Entre os que evoluíram a óbito, o período variou de seis a 73 dias, com a média de 27,8 dias. Dados menores se comparados a um estudo que aponta uma média de 30,1 dias de internação hospitalar para os pacientes que foram de alta e 29,8 dias para os que incorreram ao óbito ${ }^{(20)}$. Já o tempo médio de internação na Unidade de Terapia Intensiva dos pacientes estudados, foi de 10,5 dias, variando entre um e 40 dias. Tempo maior em relação aos dados obtidos em outro estudo que apresentou em média 6,2 $\operatorname{dias}^{(21)}$.

Diversos autores relatam correlação entre a hipertensão arterial sistêmica com o risco de ruptura de aneurismas, mesmo sem consenso na literatura que seja bem definido ${ }^{(22)}$, nesta casuística $75 \%$ dos pacientes que foram operados eram hipertensos.

Das sequelas apresentadas nos pacientes analisados, 39,2\% tiveram hemiparesia, 17,8\% déficits cognitivos - em todos os graus de comprometimento, $10,7 \%$ diminuição da memória, 10,7\% alguma alteração de marcha, $10,7 \%$ ptose palpebral, $7,1 \%$ estrabismo, $7,1 \%$ afasia de expressão e 7,1\% disfagia. Estudo realizado no Hospital Nossa Senhora da Conceição, com o intuito de identificar quais sequelas apresentadas em pacientes com hemorragia subaracnóidea por ruptura de aneurisma intracraniano, também apontou que as sequelas neurológicas apareceram em maior prevalência $(54,0 \%)$, seguida por alterações cognitivas $(45,9 \%)$, déficits motores $(31,1 \%)$, sequelas comportamentais e outras alterações $(6,9 \%)^{(14)}$.

\section{Conclusões}

Prevaleceram pacientes adultos jovens, do gênero feminino, que chegaram à Instituição transferidos de outro hospital, com aneurismas únicos, rotos, em circulação anterior, evoluindo sem sequelas e com uma baixa mortalidade.

\section{Referências}

1. Holanda L. Hemorragia meníngea. In: Manual de neurocirurgia. $2^{a}$. ed. São Paulo: Fundo BYK; 1995. p. 87-95.

2. Zhang J, Claterbuck RE. Molecular genetics of human intracranial aneurysms. Int J Stroke. 2008; 3(4):272-87.

3. Cardozo Junior LCM, Barros BP, Holanda MFC. Fatores de risco em pacientes com aneurismas intracranianos atendidos em um hospital de referência de Belém-PA. J Bras Neurocir. 2011; 22(3):95-9.

4. Peres CMA, Carrera AB, Daniel JW, Cannoni LF, Carvalho RRD. Hemorragia subaracnóidea por aneurisma intracraniano. Arq Bras Neurocir. 1998; 17(1):25-30.

5. The International Study of Unruptured Intracranial Aneurysms Investigators. Unruptured intracranial aneurysm, risks of surgical intervention. N Engl J Med. 1998; 339(24):1725-33.

6. Brock RS. Hemorragia subaracnóidea espontânea. In: Adoni T, Brock RS. Neurologia e neurocirurgia. São Paulo: Atheneu; 2008. p. 379-98.

7. Bonilha L, Marques EL, Carelli EF, Fernandes YB, Cardoso AC, Maldaum MVM, et al. Risk factors and outcome in 100 patients with aneurysmal subarachnoid hemorrhage. Arq Neuropsiquiatr. 2001; 59(3B):676-80.

8. Passos Filho P. Aneurisma cerebral: doença que causa derrame pode não apresentar sintomas. Folha Universal. 25 nov. 2007. Medicina \& Saúde. p.10.

9. Bederson JB, Connolly ES Jr, Batjer HH, Dacey RG, Dion JE, Diringer MN, et al. Guidelines for the management of aneurysmal subarachnoid hemorrhage: a statement for healthcare professionals from a special writing group of the Stroke Council, American Heart Association. Stroke. 2009; 40(3):994-1025.

10. Raymond J. Incidental intracranial aneurysms: rationale for treatment. Curr Opin Neurol. 2009; 22(1):96-102.

11. Amato ACM, Santos RV, Amato SJTA. Manual do médico generalista na era do conhecimento. $2^{\mathrm{a}}$ ed. São Paulo: Roca; 2014. $688 \mathrm{p}$.

12. Costa Filho A. Santa Casa de Misericórdia de São Paulo e a neurocirurgia. Arq Med Hosp Fac Cienc Med Santa Casa São Paulo. 2002; 47(2):61-71.

13. Diccini S, Torres AC, Silveira DAP. Intervenções de enfermagem no pré e pós-operatório em pacientes submetidos a tratamento neurocirúrgico. In: Koizumi MS, Diccini S. Enfermagem em neurociência: fundamentos para a prática clínica. São Paulo: Atheneu; 2006. p. 183-206.

14. Guaresi JR, Iung TC, Branco LTO, Medeiros MS, Sakae TM. Sequelas em pacientes com hemorragia subaracnóidea por ruptura de aneurisma intracraniano. ACM Arq Catarin Med. 2011; 40(2):35-40.

15. Carvalho PR, Silva MN, Rodrigues Júnior JC, Barbosa BA, Oliveira EM, Ner B, et al. Microcirurgia para aneurismas intracranianos múltiplos: série de 29 casos. Arq Bras Neurocirur. 2014; 33(2):89-94.

16. Araújo OF, Sousa CLM, Muniz MV, Oliveira AB, Freire Neto NG, Sousa EPD. Diagnósticos de enfermagem e proposta de intervenções ao paciente com aneurisma cerebral. Comun. Ciênc Saúde. 2014; 25(1):25-34. 
17. Mhurchu CN, Anderson C, Jamrozik K, Hankey G, Dunbabin D; Australasian Cooperative Research on Subarachnoid Hemorrhage Study (ACROSS) Group. Hormonal factors and risk of aneurismal subarachnoid hemorrhage: an international population-based, case-control study. Stroke. 2001; 32(3):606-12.

18. De Rooji, NK, Linn FH, Vande Plas JA, Algra A, Rikel GJ. Incidence of subarachmois: a systtematic review with emphasis on region, age, gender and time trends. J Neurol Neurosurg Neur Psychiatry. 2007; 78(12):1365-72.

19. Brasil. Ministério da Saúde. Secretaria de Atenção à Saúde. Departamento de Atenção Especializada e Temática Coordenação Geral de Média e Alta Complexidade. Informações sobre Ações da Epilepsia. 25 de setembro de 2015. Nota informativa $n^{\circ} 131 / 2015$.

20. Martins PA, Goulart RN, Marques MOT, Ghizoni E. Hemorragia subaracnóidea aneurismática: análise da evolução dos pacientes internados em um hospital de Tubarão. ACM Arq Catarin Med. 2012; 41(4):19-25.

21.Lins AC, Pontes GO, Damian MM. Infecções hospitalares em pacientes submetidos à clipagem de aneurisma internados na Unidade de Terapia Intensiva do Hospital Universitário do hospital Getúlio Vargas na cidade de Manaus - AM. J Bras Neurocir. 2012; 23(4):281-7.

22. Shea AM, Reed SD, Curtis LH, Alexander Michael J, Villani JJ, Schulman KA. Characteristics of nontraumatic subarachnoid hemorrhage in the United States in 2003. Neurosurgery. 2007; 61(6):1131-7.

Artigo recebido: 07/12/2016

Artigo aprovado: 10/02/2018 